\title{
Every Gauge Orbit \\ Passes Inside the Gribov Horizon
}

\author{
Gianfausto Dell'Antonio ${ }^{1, \star}$ and Daniel Zwanziger ${ }^{2, \star \star}$ \\ 1 Dipartimento di Matematica, Università di Roma La Sapienza, I-00185 Roma, Italy \\ 2 Physics Department, New. York University, New York, NY 10003, USA
}

Received June 25, 1990; in revised form October 31, 1990

\begin{abstract}
The $L^{2}$ topology is introduced on the space of gauge connections $\mathbf{A}$ and a natural topology is introduced on the group of local gauge transformations GT. It is shown that the mapping $G T \times \mathbf{A} \rightarrow \mathbf{A}$ defined by $A \rightarrow A^{g}=g^{*} A g+g^{*} d g$ is continuous and that each gauge orbit is closed. The Hilbert norm of the gauge connection achieves its absolute minimum on each gauge orbit, at which point the orbit intersects the region bounded by the Gribov horizon.
\end{abstract}

\section{Introduction}

In a gauge theory, configurations which are related by gauge transformations are physically equivalent. Because gauge transformations are non-linear, the physical configuration space, which is the space of connections modulo gauge transformations, is a non-linear space of which we lack an explicit description. Moreover, in Euclidean quantization of a gauge theory we are instructed to integrate over the physical configuration space, so it seems worthwhile to gather what information we can about it.

In the work of Gribov [1], who first pointed out the difficulty, it was conjectured that the physical configuration space may be identified with those connections $A$ which satisfy two conditions: (1) transversality: $\partial \cdot A=0$, and (2) positivity of the Faddeev-Popov operator $K[A] \equiv-D(A) \cdot \partial \geqq 0$.

These two conditions define the Gribov region $\Omega$, whose boundary is called the (first) Gribov horizon. It was subsequently pointed out [2] that the two conditions which define $\Omega$ are the conditions for a local minimum of the Hilbert norm on each gauge orbit. More specifically, if

$$
A^{g} \equiv g^{*} A g+g^{*} d g
$$

$\star$ CNR, GNFM

$\star \star$ Research supported in part by the National Science Foundation under grant no. PHY 87-15995 
is the gauge-transform of $A(x)$ by the local gauge transformation $g(x)$, then, for fixed $A$, as $g$ varies over the group of local gauge transformations, one finds by an elementary calculation that the Hilbert norm square

$$
S_{A}[g] \equiv\left\|A^{g}\right\|^{2} \equiv \int d \mu(x)\left|A^{g}\right|^{2}
$$

is stationary at $\partial \cdot A^{g}=0$, and moreover at a local minimum the Faddeev-Popov operator is positive, $K\left[A^{g}\right] \geqq 0$. Thus, the set made up of the local minima of the Hilbert norm on each gauge orbit lies in $\Omega$. Conversely, every interior point $A$ of $\Omega$ is a local minimum of the Hilbert norm on the gauge orbit through $A$. It is also known that $\Omega$ is convex and bounded in every direction [2], and that (if Euclidean space is a torus) it is contained inside the ellipsoid $\int d k|a(k)|^{2} / k^{2} \leqq C$, where $C$ is a constant that depends on the gauge group and the dimension $D$ of space-time [3]. Gribov has proposed that confinement of gluons may be due to the restriction of the physical configuration space to $\Omega$ [1]. For these reasons it is of interest to know whether all gauge orbits do indeed intersect the Gribov region $\Omega$.

In the present article we shall show that the Hilbert norm $\left\|A^{g}\right\|$ achieves its absolute minimum on each orbit. The precise formulation of this problem leads to a natural definition of the local gauge group $G T_{1}$ as an (infinite-parameter) topological group. We show that, given $A$, there exists some local gauge transformation $h \in G T_{1}$ such that $\left\|A^{h}\right\| \leqq\left\|A^{g}\right\|$ for all local gauge transformations $g \in G T_{1}$. Moreover, since every minimum lies in $\Omega$, it follows that every gauge orbit does in fact intersect $\Omega$, at the absolute minimum of its Hilbert norm. [As discussed in the next section, the basic idea is that the topology is chosen to make the gauge transformation (1.1) well defined when the space $A$ of connections is taken to be the $L^{2}$-space. This implies that $d g$ possesses an $L^{2}$ norm, so the group of gauge transformations is contained in $H_{1}$.] The existence of the minimum on each orbit follows from the fact that every minimizing sequence $g_{n}$-i.e. such that $S_{A}\left[g_{n}\right]$ approaches its absolute minimum - contains a subsequence which converges in $G T_{1}$ to a limiting gauge transformation $h$. We remark that convergence in $G T_{1}$ does not follow from compact embedding theorems, which only provide convergence in weaker norms. We also show that for fixed $A$, the gauge transformation (1.1) is a continuous mapping from $G T_{1}$ into the space $\mathbf{A}$ of $L^{2}$ connections, and that each gauge orbit is closed in $\mathbf{A}$.

We mention a related result due to Semenov-Tyan-Shanskii, and Franke [4] for which details are provided in [5]. The set $A$ of points $B$ which are absolute minima on each gauge orbit is convex, and moreover $A$ is a proper subset of the Gribov region $\Omega$. Thus $\Omega$ is not a fundamental modular region. The elements of the difference set $\Omega-\Lambda$ are relative minima that are not absolute minima, and they are Gribov copies that lie inside the first Gribov horizon. On the other hand, one would expect that the set $\Lambda$ of absolute minima on each gauge orbit would constitute a fundamental modular region [6]. For there does not appear to be any systematic degeneracy which would cause the absolute minimum to be achieved at more than one point on an orbit.

Our results are stated in Sect. 2A and proved in Sect. 2B. The extension to Euclidean space of infinite volume is given in Sect. 3.

\section{A. Statement}

Let $N$ be a smooth $d$-dimensional compact Riemannian manifold, and let $d \mu$ be a measure on $N$, absolutely continuous relative to Lebesgue measure, with bounded 
density; without loss of generality we assume that the total mass of $\mu$ is one. Let $\mathrm{G}$ be an $N$-dimensional, fundamental unitary representation of a compact nonabelian group, and denote by $G$ its Lie algebra in the adjoint representation. Let $E_{0}$ be the set of smooth $C^{\infty}$-valued one-forms, with values in $N \times N$ complex matrices. We denote by $E$ the Hilbert space which is obtained completing $E_{0}$ in the norm induced by the scalar product

$$
M_{1}, M_{2} \in E_{0}: \quad\left(M_{1}, M_{2}\right) \equiv N^{-1} \int_{N} \mu(d x) \operatorname{Tr}\left(M_{1}(x)^{*} M_{2}(x)\right),
$$

where Tr includes the trace for $N \times N$ matrices and the scalar product at $x$ of oneforms as given by the Riemann structure; we write $\|M\|$ for $(M, M)^{1 / 2}$.

We denote by $\mathbf{A}_{0}$ the set of smooth $G$-valued one-forms, and by $\mathbf{A}$ its closure in $E$. Let $G T_{0}$ be the space of smooth gauge transformations, i.e. smooth maps from $N$ to $\mathrm{G}$; it is a subset of the smooth maps from $N$ to $N \times N$ matrices. We shall denote by $G T$ its closure in $L^{2}(N, \mu)^{N \times N}$ and by $G T_{1}$ its closure in $H_{1} \equiv\left\{f \in L^{2}(N, \mu)^{N \times N} \mid \nabla f \in L^{2}(N, \mu)^{N \times N \times d}\right\}$. We denote by $\|\cdot\|$ and by $\|\cdot\|_{1}$ the corresponding norms, i.e. $\|f\|^{2}=\int_{N}|f|(x) \mu(d x),\|f\|_{1}^{2}=\|f\|^{2}+\|\nabla f\|^{2}(|f|$ is the trace norm of the matrix $f$ ).

Since $N$ is compact, $G T_{1}$ is a compact subset of $G T$.

Notice that, if $g \in G T_{0}, g(x)$ is unitary for all $x \in N$, and one has for every $M \in E$,

$$
\|g M\|=\|M g\|=\|M\| \text {. }
$$

Denote by $\sigma^{0}(A)$ the orbit of $G T_{0}$ through $A$,

$$
\sigma^{0}(A) \equiv\left\{A^{g} \mid g \in G T_{0}\right\} \text {. }
$$

Denote by $\sigma(A)$ the closure of $\sigma^{0}(A)$ in $\mathbf{A}$. We shall prove the propositions which follow.

Proposition 1. GT $T_{1}$ is a topological group and the map (1.1) extends to a continuous action of $G T_{1}$ on $E$. Moreover, $\sigma(A)=\left\{A^{g} \mid g \in G T_{1}\right\}$, i.e. the closure of the orbit is the orbit of the closure.

Remark 1. For some compact Lie groups, the group of local gauge transformations $G T_{0}$ is composed of disjoint sectors characterized by topological properties, for example a winding number. This is not true in general of $G T_{1}$. In particular there are local gauge transformations in $G T_{1}$ which do not have a well-defined winding number. Consequently, there is not in general an absolute minimum in each topological sector.

Remark 2. For $A \in \mathbf{A}$, consider on $G T_{0}$ the functional

$$
S_{A}(g) \equiv\left\|A^{g}\right\|^{2} \text {. }
$$

By Proposition 1, this functional extends to a continuous functional on $G T_{1}$. It is easy to verify that $S_{A}(g)$ attains a (local) minimum at the identity of $G T_{1}$ only if a) $\partial_{\mu} A_{\mu}=0$ weakly,

(b) for every $w \in W_{0}$, where $W_{0}$ is the space of $C^{\infty}$ maps from $N$ to $G$, one has

$$
(d w, d w)-([w, A], d w) \geqq 0,
$$

where $[w, A]^{a}(x) \equiv f^{a b c} w^{b}(x) A^{c}(x)$, and $f^{a b c}$ are the structure constants of the group G. If $A$ has further smoothness properties (depending on the dimension of the manifold $N)$, the bilinear form $\left(d w_{1}, d w_{2}\right)-\left(\left[w_{1}, A\right], d w_{2}\right)$ is closable [and sym- 
metric of a) is satisfied] and therefore, whenever positive it defines a (positive) selfadjoint operator, formally given in local coordinates by $-\Delta-\left[A_{\mu}, \partial_{\mu}\right]$.

This is the Faddeev-Popov operator $K[A]$.

We shall denote by $\Omega$ the subset of $\mathbf{A}$ for which a) and b) holds. From its definition it follows that $\Omega$ is closed in $\mathbf{A}$. [Condition a) can be expressed by the statement that $A$ is orthogonal in $\mathbf{A}$ to every gradient vector field.]

Proposition 2. Every orbit of $G T_{1}$ intersects $\Omega$ at least once.

Indeed, since $S_{A}(g)$ is continuous in $G T_{1}$, it follows by a) and b), that every orbit intersects $\Omega$ at the absolute minimum of $S_{A}(g)$, whose existence is asserted in the following statement (which then implies Proposition 2).

Proposition 3. For every sequence $\left\{g_{n}\right\}$ which is a minimizing sequence for the functional $S_{A}(g)$, there is a subsequence $g_{n}^{\prime}$ which converges in the topology of $G T_{1}$. Let $h \in G T_{1}$ be the limit. The sequence $A^{g_{n}^{\prime}}$ converges in the topology of $\mathbf{A}$ to $A^{h}$. As a consequence, on every orbit, the continuous functional $S_{A}(g)$ attains its absolute minimum.

Remark 1. Notice that convergence is claimed in the topology of $G T_{1}$ and not in a weaker topology. The latter would be a simple consequence of compact embedding theorems.

Remark 2. Instead of $\Omega$, one could consider the subset $\Lambda$ defined by

$$
\Lambda \equiv\left\{A \in \Omega \mid S_{A}(g) \text { attains the absolute minimum at the identity of } G T_{1}\right\} .
$$

In view of Proposition 3, $\Lambda$ is also intersected at least once by every orbit. From Proposition 1 and the continuity of $S_{A}(g)$ in $\mathbf{A}$, it follows that $\Lambda$ is also closed in $\mathbf{A}$. (This follows from arguments given in [5].) However, $\Lambda$ does not have a simple characterization in terms of differential operators, and we have no estimate on its size. Still one can prove $[4,5]$ that $\Lambda$ is convex and that the inclusion $\Omega \supset \Lambda$ is strict. Moreover, since $\Lambda$ is closed it follows that the difference set $\Omega^{0}-\Lambda$ is open, where $\Omega^{0}$ is the interior of $\Omega$.

Remark 3. Conditions a) and b) are meaningful for every distribution-valued oneform. One can therefore consider instead of $\Omega$, the subset $\Omega^{\prime}$ of distribution-valued connections on $N$ which satisfy a) and b). For such distributions one can define the map (1.1) for all $g \in G T_{0}$. However, the functional $S_{A}(g)$ is not defined in general for $A \in \Omega^{\prime}$ [although formally $S_{A}(g)-S_{A}\left(g^{\prime}\right)$ can be defined]. As a consequence the enlarged set $\Omega^{\prime}$ does not appear as a set of local minima of a functional defined on $G T_{0}$. Nevertheless, about $\Omega^{\prime}$ one knows that it is convex and one can establish an a-priori Sobolev bound on the elements of $\Omega^{\prime}[3]$.

\section{B. Proofs}

We shall now prove Propositions 1 and 3. Proposition 2 is a consequence of Proposition 3. The following simple lemmas will be useful.

Lemma I. If $\lim _{n, m \rightarrow \infty}\left\|d g_{n}-d g_{m}\right\|=0$ and $\left\|d g_{n}\right\|<c \forall n$, there is a subsequence $g_{n}^{\prime}$ such that $\lim _{n, m \rightarrow \infty}\left\|g_{n}^{\prime, m \rightarrow g_{m}^{\prime}}\right\|_{1}=0$.

Proof. Write $g=g^{(1)}+\langle g\rangle$, where the matrix $\langle g\rangle$ is the mean of $g(x)$ over $N$. From $\left\|d g_{n}^{(1)}\right\|\left\langle c \forall n\right.$ and $\left\langle g_{n}^{(1)}\right\rangle=0$ it follows by Poincaré's lemma that $\left\|d g_{n}^{(1)}-d g_{m}^{(1)}\right\| \rightarrow 0$ 
implies $\left\|g_{n}^{(1)}-g_{m}^{(1)}\right\| \rightarrow 0[7]$. Since $\left|\left\langle g_{n}\right\rangle\right| \leqq 1$, there exists a subsequence $g_{n}^{\prime}$ such that $\left\langle g_{n}^{\prime}\right\rangle$ converges. Therefore, $\left\|g_{n}^{\prime}-g_{m}^{\prime}\right\| \rightarrow 0$.

Lemma II. Let $\left\{g_{n}\right\}, B$ be maps from $N$ to $D \times D$ matrices, with $\left|g_{n}(x)\right|<c$ for all $x \in N$, and $\|B\|^{2} \equiv \int_{N}|B(x)|^{2} \mu(d x)<+\infty$. Assume that $\left\|g_{n}-g_{m}\right\| \rightarrow 0$. Then $\left\|g_{n} B-g_{m} B\right\| \rightarrow 0$.

Proof. For every $\varepsilon>0$ one can find a $\mu$-measurable subset $K(\varepsilon)$ of $N$ and a positive constant $M_{\varepsilon}$ such that $\int_{K(\varepsilon)}|B(x)|^{2} \mu(d x)<\varepsilon$ and $|B(x)|<M_{\varepsilon} \forall x \in K^{c}(\varepsilon)$. Then

$$
\begin{aligned}
\int_{N}\left|g_{n} B-g_{m} B\right|^{2} \mu(d x) & \leqq 4 c^{2} \int_{K(\varepsilon)}|B(x)|^{2} \mu(d x)+M_{\varepsilon}^{2} \int_{K^{c}(\varepsilon)}\left|g_{n}(x)-g_{m}(x)\right|^{2} \mu(d x) \\
& \leqq 4 c^{2} \varepsilon+M_{\varepsilon}^{2}\left\|g_{n}-g_{m}\right\|^{2} .
\end{aligned}
$$

Since $\varepsilon$ is arbitrary and $\left\|g_{n}-g_{m}\right\| \rightarrow 0$ by assumption, for every $\delta>0$ one can find $N(\delta)$ such that $\left\|g_{n} B-g_{m} B\right\|<\delta$ if $n, m>N(\delta)$.

Proof of Proposition 1. To prove that $G T_{1}$ is a topological group it is sufficient to verify that the action of $G T_{0}$ on $G T_{1}$ given by $g \rightarrow g h$ is continuous in $g$ in the topology of $G T_{1}$. To prove this assume that

$$
\lim _{n \rightarrow \infty}\left\|g_{n}-g\right\|_{1}=0, \text { for } g_{n} \in G T_{0} \text { and } g \in G T_{1} \text {. }
$$

We want to prove that for every $h \in G T_{1}, \lim _{n \rightarrow \infty}\left\|g_{n} h-g h\right\|_{1}=0$. We have

$$
\left\|g_{n} h-g h\right\|_{1}^{2}=\left\|d\left(g_{n} h\right)-d(g h)\right\|^{2}+\left\|g_{n} h-g_{m} h\right\|^{2} .
$$

The first term is estimated by Leibnitz's rule and the triangle inequality. The conclusion follows then from Lemma I.

To prove that (1.1) is a continuous mapping and that $\sigma_{A}$ is closed in $\mathbf{A}$ it is sufficient to prove:

a) If $g_{n}, h \in G T_{1}$. and $\lim _{n \rightarrow \infty}\left\|g_{n}-h\right\|_{1}=0$, then $\lim _{n \rightarrow \infty}\left\|A^{g_{n}}-A^{h}\right\|=0$.

b) If $\lim _{n, m \rightarrow \infty}\left\|A^{g_{n}}-A^{g_{m}}\right\|=0$, then one $\underset{n \rightarrow \infty}{\text { can }}$ find $h \in G T_{1}$ such that

$\lim _{n \rightarrow \infty}\left\|A^{g_{n}}-A^{h}\right\|=0$, and moreover, $\exists$ a subsequence (still denoted by $g_{n}$ ) such that $\lim _{n \rightarrow \infty}\left\|g_{n}-h\right\|_{1}=0$.

To prove a) one has, using the triangle inequality,

$$
\begin{aligned}
\left\|A^{g_{n}}-A^{h}\right\|= & \left\|g_{n}^{*} A g_{n}-h^{*} A h+g_{n}^{*} d g_{n}-h^{*} d h\right\| \\
\leqq & \left\|g_{n}^{*} A\left(g_{n}-h\right)\right\|+\left\|\left(g_{n}^{*}-h^{*}\right) A h\right\| \\
& +\left\|\left(g_{n}^{*}-h^{*}\right) d h\right\|+\left\|g_{n}^{*}\left(d g_{n}-d h\right)\right\| \\
\leqq & 2\left\|A\left(g_{n}-h\right)\right\|+\left\|\left(g_{n}^{*}-h^{*}\right) d h\right\|+\left\|g_{n}-h\right\|_{1} .
\end{aligned}
$$

The conclusion follows now from Lemma II.

To prove b), assume that $\lim _{n \rightarrow \infty}\left\|A^{g_{n}}-B\right\| \rightarrow 0$. Then

$$
\left\|d g_{n}\right\|=\left\|g_{n}^{*} d g_{n}\right\| \leqq\|B\|+\left\|g_{n}^{*} A g_{n}\right\|+R_{n}=\|B\|+\|A\|+R_{n},
$$

where $\lim _{n \rightarrow \infty} R_{n}=0$. Therefore, $\left\|d g_{n}\right\|<C$ uniformly in $n$ for some constant $C$, and since $\left|\left\langle g_{n}\right\rangle\right| \leqq 1$, it follows as in the proof of Lemma I that $g_{n}$ has a subsequence 
(which we still denote by $g_{n}$ ) which converges in $G T$ to $h \in G T_{1}$. Arguing as in the proof of Lemma II, one has

But then

$$
\lim _{n \rightarrow \infty}\left\|g_{n}^{*} A g_{n}-h^{*} A h\right\|=0
$$

or

$$
\lim _{n \rightarrow \infty}\left\|g_{n}^{*} d g_{n}-B+h^{*} A h\right\|=0
$$

$$
\lim _{n \rightarrow \infty}\left\|d g_{n}-g_{n} B+g_{n} h^{*} A h\right\|=0,
$$

It follows from Lemma I that

$$
\lim _{n, m \rightarrow \infty}\left\|d g_{n}-d g_{m}\right\|=0 .
$$

and this, together with $g_{n} \rightarrow h$ in $G T$ implies $\lim _{n, m \rightarrow \infty}\left\|g_{n}-g_{m}\right\|_{1}=0$, and therefore, $g_{n} \rightarrow h$ in $G T_{1}$.

Since $\left|g_{n}(x)\right|=1$ for all $x$, it follows that

so that

$$
\left\|g_{n}^{*} d g_{n}-h^{*} d h\right\| \leqq\left\|d g_{n}-d h\right\|+\left\|g_{n}^{*} d h-h^{*} d h\right\|
$$

But then

$$
\lim _{n \rightarrow \infty}\left\|g_{n}^{*} d g_{n}-h^{*} d h\right\|=0
$$

$$
\left\|h^{*} d h+h^{*} A h-B\right\| \leqq \lim _{n \rightarrow \infty}\left[\left\|g_{n}^{*} d g_{n}-h^{*} d h\right\|+\left\|g_{n}^{*} d g_{n}-B+h^{*} A h\right\|\right]=0,
$$

so that $B=A^{h}$.

Proof of Proposition 3. Let $g_{n} \in G T_{1}$ be a minimizing sequence for $S_{A}(g) \equiv\left\|A^{g}\right\|^{2}$, so that

$$
\lim _{n \rightarrow \infty} S_{A}\left(g_{n}\right)=m_{A} \equiv \inf _{g \in G T_{1}} S_{A}(g)
$$

One has

so that

$$
\left[S_{A}\left(g_{n}\right)\right]^{1 / 2}=\left\|g_{n}^{*} A g_{n}+g_{n}^{*} d g_{n}\right\|
$$

$$
\begin{aligned}
\left\|d g_{n}\right\| & =\left\|g_{n}^{*} d g_{n}\right\| \leqq\left\|g_{n}^{*} A g_{n}\right\|+\left[S_{A}\left(g_{n}\right)\right]^{1 / 2} \\
& =\|A\|+\left[S_{A}\left(g_{n}\right)\right]^{1 / 2} \leqq 2\|A\| .
\end{aligned}
$$

Therefore, $\left\|d g_{n}\right\|$ is uniformly bounded. From Lemma $\mathrm{I}$ it follows that there is a constant $C$ and a subsequence (also denoted by $\left\{g_{n}\right\}$ ) which satisfies $\left\|g_{n}\right\|_{1} \leqq C$ for all $n$ and converges in the topology of $G T$ to an element $h \in G T_{1}$, which also satisfies $\|h\|_{1} \leqq C[7]$. Therefore, $B \equiv A^{h}$ is well defined.

Define

$$
\Delta_{n} \equiv S_{A}\left(g_{n}\right)-S_{A}(h)=S_{B}\left(u_{n}\right)-S_{B}(I),
$$

where $u_{n} \equiv h^{*} g_{n}$. We have

$$
\begin{aligned}
\Delta_{n} & =\left\|u_{n}^{*} B u_{n}+u_{n}^{*} d u_{n}\right\|^{2}-\|B\|^{2} \\
& =2 \operatorname{Re}\left(B\left(u_{n}-1\right), d u_{n}\right)+2 \operatorname{Re}\left(B, d u_{n}\right)+\left\|d u_{n}\right\|^{2} .
\end{aligned}
$$

Notice that $h^{*} g_{n} \in G T_{1}$ and $\left\|h^{*} g_{n}-I\right\|=\left\|g_{n}-h\right\|$, so that $u_{n}=h^{*} g_{n} \rightarrow I$ in $E$. 
It follows from Lemma II that

$$
\lim _{n \rightarrow \infty}\left\|B\left(u_{n}-I\right)\right\| \rightarrow 0,
$$

and therefore, the first term in (2.6) converges to zero.

To estimate $\left|\left(B, d u_{n}\right)\right|$, notice that for every $\varepsilon>0$, one can find $\underline{B}$ which is of class $C^{1}$ and such that $\|B-\underline{B}\|<\varepsilon$. We have

$$
\left|\left(B, d u_{n}\right)\right| \leqq 2 \varepsilon C+\left|\left(\underline{B}, d u_{n}\right)\right|,
$$

since $\left\|d u_{n}\right\|=\left\|d\left(h^{*} g_{n}\right)\right\| \leqq\|d h\|+\left\|d g_{n}\right\| \leqq 2 C$.

It suffices, therefore, to prove that

Integrating by parts

$$
\lim _{n \rightarrow \infty}\left|\left(\underline{B}, d u_{n}\right)\right| \rightarrow 0 .
$$

$$
\lim _{n \rightarrow \infty}\left(\underline{B}, d u_{n}\right)=\left(\underline{B}, d\left(u_{n}-I\right)\right)=\left(\delta \underline{B},\left(u_{n}-I\right)\right) \leqq\|\delta \underline{B}\|\left\|u_{n}-I\right\| \rightarrow 0,
$$

where $\delta=* d^{*}$.

For every $\varepsilon>0$ one has then, for some positive constant $C_{3}$,

$$
0 \geqq \lim _{n \rightarrow \infty} S_{A}\left(g_{n}\right)-S_{A}(h) \geqq C_{3} \varepsilon+\limsup _{n \rightarrow \infty}\left\|d u_{n}\right\|^{2} .
$$

Since $\varepsilon$ is arbitrary and $\left\|d u_{n}\right\|^{2} \geqq 0$, we conclude

$$
\begin{gathered}
\lim _{n \rightarrow \infty} S_{A}\left(g_{n}\right)-S_{A}(h)=0, \\
\lim _{n \rightarrow \infty}\left\|d u_{n}\right\|=0 .
\end{gathered}
$$

We conclude that $S_{A}(g)$ achieves its absolute minimum at $h$.

Notice that from (2.10) one also derives

$$
\lim _{n \rightarrow \infty}\left\|d g_{n}-d h\right\|=0 \text {. }
$$

We have already proved that there is a subsequence $g_{n}^{\prime}$ which converges in $L^{2}(N, \mu)^{N \times N}$ to $h$. Therefore, $g_{n}^{\prime}$ converges to $h$ in $G T_{1}$. From this and the continuity of the action of $G T_{1}$ on $\mathbf{A}$ one concludes that $A^{g_{n}}$ converges in $\mathbf{A}$ to $A^{h} \in \Omega$.

\section{Extension to Non-Compact Manifolds}

We now extend this analysis to the case in which $N$ is not compact and $d \mu$ is not of finite mass. We give the relevant definition and sketch the modifications needed in the proofs. The spaces $E$ and $\mathbf{A}$ are defined as before. For $G T_{0}$ we take the set of those $C^{\infty}$ maps from $N$ to $G$ which take the value $I$ outside some compact set in $N$. We denote by $G T$ the completion of $G T_{0}$ in $L_{\text {loc }}^{2}(N, \mu)^{N \times N}$ and by $G T_{1}$ the completion of $G T_{0}$ in the topology induced by the seminorms

$$
\|u\|^{(m)} \equiv \int_{N}|d u|^{2} \mu(d x)+\int_{K m}|u(x)|^{2} \mu(d x),
$$

where $K_{m}$ is an increasing sequence of compact subsets which invade $N$.

One has then

Proposition 4. The statements in Propositions 1-3 hold also for the case in which $N$ is not compact. 
Sketch of proof. In the proof of Propositions 1-3, all the steps and estimates which only involve the norm $\|\cdot\|$ also hold now without change [compactness of $N$ is never used there and convergence in $L_{\mathrm{loc}}^{2}$ together with $\left|g_{n}(x)\right|=1$ for all $x$ in $N$ is sufficient for all estimates].

The estimates which involved both the norm $\|\cdot\|$ and the norm $\|\cdot\|_{1}$ required compact embeddings, and fail if $N$ is not compact. However, they still hold in every compact subset $K$ and this is sufficient in view of the following "localization" procedure, which we give explicitly for the extension of Proposition 3 to the noncompact case.

Let $g_{n} \in G T_{1}$ be a minimizing sequence for $S_{A}(g)$, so that

As before one has

$$
\lim _{n \rightarrow \infty} S_{A}\left(g_{n}\right)=m_{A}=\inf _{g \in G T_{1}} S_{A}(g) \text {. }
$$

$$
\left\|d g_{n}\right\| \leqq 2\|A\| \quad \text { for all } n,
$$

and therefore, for every compact $K$ one has

$$
\int_{K}\left|d g_{n}\right|^{2} \mu(d x) \leqq 2\|A\|_{2},
$$

so that one can find a subsequence $g_{n}^{\prime}$ such that

$$
\int_{K}\left|g_{n}^{\prime}-g_{m}^{\prime}\right| \mu(d x) \rightarrow 0 \text { when } \quad m, n \rightarrow \infty .
$$

Using the countable sequence of increasing compact sets $K_{n}$ and passing to subsequences one can find a subsequence, still denoted by $g_{n}$, which converges in $L_{\text {loc }}^{2}(N, \mu)^{N \times N}$ to an element $h$ in $L_{\text {loc }}^{2}(N, \mu)^{N \times N}$. Moreover, since $\left|g_{n}(x)\right|=1$ for every $x$ in $N$, for each compact $K$ there exists a version of $h$ for which $|h(x)|=1$ for all $x$ in $K$. Since (3.1) holds uniformly in $n$ one has $\int_{N}|d h|^{2} \mu(d x) \leqq 2\|A\|^{2}$ so that $h \in G T_{1}$.

By definition of $m_{A}$ one has

Let $B \equiv A^{h}$, and $u_{n} \equiv h^{*} g_{n}$.

$$
\lim _{n \rightarrow \infty}\left[S_{A}\left(g_{n}\right)-S_{A}(h)\right] \leqq 0 .
$$

As in (2.7),

$$
S_{A}\left(g_{n}\right)-S_{A}(h)=S_{B}\left(u_{n}\right)-S_{B}(I)=2 \operatorname{Re}\left[B\left(u_{n}-I\right), d u_{n}\right]+2 \operatorname{Re}\left(B, d u_{n}\right)+\left\|d u_{n}\right\|^{2} .
$$

From Lemma II it follows that $u_{n}-I$ converges to zero in $L_{\text {loc }}^{2}$ and $\left\|d u_{n}\right\|<C_{1} \forall n$. Choose $\varepsilon>0$. Since $B \in \mathbf{A}$, one can find a compact set $K \in N$ and a connection $\underline{B}$ of class $C^{1}$ with support in $K$ such that $\|B=\underline{B}\|<\varepsilon$.

From Lemma II one has, since $\left\|d u_{n}\right\| \leqq 2\|\bar{A}\|$,

One has, moreover,

$$
\lim _{n \rightarrow \infty}\left|2 \operatorname{Re}\left[B\left(u_{n}-I\right), d u_{n}\right]\right| \leqq 2 C_{2} \varepsilon .
$$

so that

$$
\left|2 \operatorname{Re}\left(B, d u_{n}\right)\right| \leqq 2 C_{1} \varepsilon+\left|2 \operatorname{Re}\left(\delta \underline{B},\left(u_{n}-I\right)\right)\right|
$$

$$
\lim _{n \rightarrow \infty}\left|2 \operatorname{Re}\left(B, d u_{n}\right)\right| \leqq 2 C_{1} \varepsilon .
$$

We conclude from (3.3) that

$$
0 \geqq \lim _{n \rightarrow \infty}\left[S_{A}\left(g_{n}\right)-S_{A}(h)\right] \geqq-2 \varepsilon\left(C_{1}+C_{2}\right)+\limsup _{n \rightarrow \infty}\left\|d u_{n}\right\|^{2} .
$$


Since $\varepsilon$ is arbitrary and $\left\|d u_{n}\right\|^{2} \geqq 0$ we conclude as in the compact case

$$
\begin{gathered}
\lim _{n \rightarrow \infty} S_{A}\left(g_{n}\right)=S_{A}(h), \\
\lim _{n \rightarrow \infty}\left\|d u_{n}\right\|=0 .
\end{gathered}
$$

From (3.4) it follows that $S_{A}(h)=\inf _{g \in G T_{1}} S_{A}(g)$ and therefore, the function $S_{A}$ attains its minimum on $G T_{1}$. Moreover, considering the subsequence $g_{n}^{\prime}$ for which $u_{n}^{\prime}$ converges to $I$ in $L_{\text {loc }}^{2}$, it follows from (3.5) that the sequence $u_{n}^{\prime}$ converges to $I$ in $G T_{1}$ and therefore, $g_{n}^{\prime}$ converges to $h$ in $G T_{1}$ and $A^{g_{n}}$ converges to $A^{h}$ in A.

Remark. The results described here can be extended also to the case in which $\mu$ is absolutely continuous with respect to Lebesgue measure but the density is only in $L_{\text {loc }}^{1}$. Let $\mu(d x)=\varrho(x) d x$. Then Propositions 1-3 still hold if one defines $G T$ as before and $G T_{1}$ to be the completion of $G T_{0}$ in the topology induced by the seminorms

$$
\|g\|^{(m)} \equiv \int_{N}|d g|^{2} \mu(d x)+\int_{H m}|g|^{2} \mu(d x),
$$

where $H_{m} \equiv\left\{x \in K_{m} \mid \varrho(x) \leqq m\right\}$ and $\left\{K_{m}\right\}$ is an increasing sequence of compact sets which invade $N$.

Acknowledgements. A previous version of the proof, which gave a weaker result, contained an error which, fortunately, was pointed out to us by Alan Sokal. We are grateful to him for this criticism without which we would not have obtained the present result, and also for suggestions and comments on a later version of this manuscript.

\section{References}

1. Gribov, V.N.: Nucl. Phys. B 139, 1 (1978)

2. Zwanziger, D.: Nucl. Phys. B 209, 336 (1982)

3. Dell'Antonio, G., Zwanziger, D.: Nucl. Phys. B 326, 333 (1989)

4. Semenov-Tyan-Shanskii, M.A., Franke, V.A.: Zap. Nauch. Sem. Leningrad. Otdeleniya Matematicheskogo Instituta im. V. A. Steklov AN SSSR, Vol. 120, p. 159, 1982 (English translation: New York: Plenum Press 1986)

5. Dell'Antonio, G., Zwanziger, D.: Proceedings of the NATO Advanced Research Workshop on Probabilistic Methods in Quantum Field Theory and Quantum Gravity, Cargèse, August 21-27, 1989, Damgaard and Hueffel (eds.), p. 107. New York: Plenum Press

6. A Euclidean functional integral based on this region has been proposed recently. Zwanziger, D. (ed.). Nucl. Phys. B 345, 461 (1990)

7. See for example Reed, M., Simon, B.: Methods of modern mathematical physics, vol. IV, p. 257, Theorem XIII.75

Communicated by A. Jaffe 
